EPV101/\#203 THE INCREASING INCIDENCE OF OBESITY AND UTERINE CANCER IN PATIENTS UNDER 55 IN ASIA AND THE UNITED STATES - WHO IS MOST AT RISK?

${ }^{1} \mathrm{C}-\mathrm{I}$ Liao, ${ }^{2} \mathrm{M}$ Richardson*, ${ }^{3} \mathrm{~K}$ Darcy, ${ }^{3} \mathrm{C}$ Tian, ${ }^{4} \mathrm{D}$ Kapp, ${ }^{5} \mathrm{~J}$ Chan. ${ }^{1} K a o h s i u n g$ Veterans General Hospital, Obstetrics and Gynecology, Kaohsiung City, Taiwan; ${ }^{2}$ University of California Los Angeles, Obstetrics and Gynecology, Los Angeles, USA; ${ }^{3}$ Gynecologic Cancer Center of Excellence, Obstetrics and Gynecology, Washington, USA; ${ }^{4}$ Stanford University School of Medicine, Department of Radiation Oncology, Stanford, USA; ${ }^{5}$ California Pacific Medical Center, Obstetrics and Gynecology, San Francisco, USA

\subsection{6/ijgc-2021-IGCS.171}

Objectives To evaluate the association between age, race, country of residence, and obesity with the rising incidence of uterine cancer in the United States (US) and Taiwan.

Methods Data were obtained from the United Statistics Cancer Statistics (USCS) program, Behavioral Risk Factor Surveillance System (BRFSS), and Taiwan Cancer Registry from 2001 to 2017. SEER*Stat and Joinpoint regression program were used for statistical analyses.

Results 560,131 White and 22,963 Asian women were identified in USCS and 13,950 women in the Taiwan Cancer Registry with uterine cancer, with an incidence rate per 100,000 of 21.9 White and 17.3 Asian women in the US and 15.0 women in Taiwan. The proportion diagnosed $<55$ years of age with uterine cancer varied by race and country of residence with $22 \%$ of Whites in the US, $40 \%$ Asians in the US and $52 \%$ of women in Taiwan $(\mathrm{P}<0.0001)$. Evaluation of annual percent changes (APC) in incidence of uterine cancer between 2001-2017 within different age groups indicated that the largest APC was observed in the women diagnosed between 35-39 years old with APC increases of $2.4 \%$ in Whites and 3.5\% in Asians in the US and 7.2\% in Taiwan $(\mathrm{P}<0.001)$. Evaluation of obesity trends in women between 2001-2017 using US BRFSS data indicated an APC of 2.4\% in Whites (range: 19-28\%) and $2.1 \%$ in Asians (range: 9$13 \%)$.

Conclusions Compared to US Whites and US Asians, Native Asians were diagnosed with uterine cancer at a younger age and rates are increasing annually. This finding may be attributed to the rise in obesity rates.

\section{EPV102/\#216 IMPLEMENTATION OF MOLECULAR STRATIFICATION IN ENDOMETRIAL CANCER THROUGH MIRNAS CHARACTERIZATION}

${ }^{1} \mathrm{AM}$ Perrone, ${ }^{2} \mathrm{G}$ Ravegnini, ${ }^{3} \mathrm{~A}$ de Leo, ${ }^{2} \mathrm{D}$ de Biase, ${ }^{2} \mathrm{~F}$ Gorini, ${ }^{1} \mathrm{E}$ de Crescenzo, ${ }^{1} \mathrm{G}$ Dondi, ${ }^{1} \mathrm{M}$ Tesei, ${ }^{1} \mathrm{M}$ di Stanislao, ${ }^{1} \mathrm{P}$ de laco. ${ }^{1}$ IRCCS Azienda Ospedaliero-Universitaria di Bologna, Division of Oncologic Gynecology, Bologna, Italy; ${ }^{2}$ University of Bologna, Pharmacy and Biotechnology, Bologna, Italy; ${ }^{3}$ University of Bologna, Dept of Experimental, Diagnostic and Specialty Medicine, Bologna, Italy

\subsection{6/ijgc-2021-IGCS.172}

Objectives Introduction. The TCGA project identified four distinct prognostic groups of endometrial carcinoma (EC) based on molecular alterations among which two are correlated with an intermediate prognosis: the MisMatch Repair deficient (MMRd) and the No Specific Molecular Profile (NSMP) groups. NSMP represents a heterogenous subset of patients frequently harboring CTNNB1 alterations and presenting distinctive clinicopathologic features comparing with the CTNNB1 non mutant ones. miRNAs are oncological key players that have not been integrated with the TCGA EC classification. The study aimed to evaluate the miRNA expression profile in EC to identify potential novel biomarkers of diagnosis and prognosis.

Methods We analyzed miRNA expression in 72 ECs specimens previously classified as MMRd (31) and NSMP (41), including 15 with CTNNB1 mutations. In the discovery step, miRNA expression profile was evaluated in 30 cases through TaqMan Advanced miRNA arrays. Subsequently, in the validation step, four miRNAs were analyzed in the total cohort of ECs by specific miRNA Assays.

Results Comparison of CTNNB1 mutant versus non-mutant ECs (irrespective of MMRd/NSMP status) in the discovery cohort showed 39 differentially expressed miRNAs. The top deregulated 4 miRNAs (miR-187, miR-325, miR-499a-3p and $5 p)$ were further validated in 72 ECs. miR-499a-3p and miR499a-5p maintained the statistical significance showing higher expression in CTNNB1 mutant ECs $(p<0.0001$, for both). Furthermore, miR-499a expression was able to identify EC subgroups with longer recurrence free survival.

Conclusions Conclusion. miR-499a may be a useful biomarker and could be integrated in the current TGCA classification scheme to better stratify EC patients

\section{EPV103/\#218 NUCLEAR FEATURES ALLOW FOR HIGHLY SENSITIVE SELECTION OF ENDOMETRIAL CARCINOMAS FOR P53 TESTING}

${ }^{1} \mathrm{E}-\mathrm{Y}$ Kang ${ }^{*},{ }^{1} \mathrm{~N}$ Wiebe, ${ }^{2} \mathrm{C}$ Aubrey, ${ }^{3,4} \mathrm{C}-\mathrm{H}$ Lee, ${ }^{5} \mathrm{M}$ Anglesio, ${ }^{6} \mathrm{D}$ Tilley, ${ }^{2} \mathrm{P}$ Ghatage, ${ }^{2} \mathrm{G}$ Nelson, ${ }^{1} \mathrm{~S}$ Lee, ${ }^{1} \mathrm{M}$ Köbel. 'University of Calgary, Department of Pathology and Laboratory Medicine, Calgary, Canada; ${ }^{2}$ University of Calgary, Department of Oncology, Division of Gynecologic Oncology, Calgary, Canada; ${ }^{3} B C$ Cancer, Department of Pathology and Laboratory Medicine, Vancouver, Canada; ${ }^{4}$ University of British Columbia, Department of Pathology and Laboratory Medicine, Vancouver, Canada; ${ }^{5}$ University of British Columbia, Department of Obstetrics and Gynecology, Vancouver, Canada; ${ }^{6}$ Alberta Health Services, Cancer Control, Calgary, Canada

\subsection{6/ijgc-2021-IGCS.173}

Objectives The World Health Organization endorses molecular subclassification of endometrial endometrioid carcinomas. Our objectives were to test the sensitivity of tumor morphology in capturing p53-abnormal (p53abn) cases and to model the impact of p53abn on changes to ESGO/ESTRO/ESP risk stratification.

Methods 292 consecutive endometrial carcinoma resections received at Foothills Medical Centre, Calgary, Canada (2019_ 2021) were retrieved and assigned to ESGO risk groups without and with p53 status. Three pathologists reviewed representative H\&Es, predicted the p53 status, and indicated whether p53 immunohistochemistry would be ordered. Population-based survival for endometrial carcinomas diagnosed 2008-2016 in Alberta was obtained from the Alberta Cancer Registry.

Results The cohort consisted mostly of grade $1 / 2$ endometrioid carcinomas (EEC12; N=218, 74.6\%). $152 \quad$ EEC12 $(52.1 \%$ overall) were stage IA and $147(50.3 \%)$ were low-risk by ESGO. The overall prevalence of p53abn and subclonal p53 was $14.5 \%$ and $8.3 \%$. The average sensitivity of predicting p53abn among observers was $83.6 \%$ and observers requested 
p53 immunohistochemistry on $39.4 \%$ with a sensitivity of $98.5 \%$ to detect p53abn (99.6\% negative predictive value). Cytologic features including tumor giant cells, smudged chromatin, cherry-red/macronucleoli, and atypical mitoses accurately predicted p53abn. In 7/292, p53abn upgraded ESGO risk groups (2 to intermediate-risk, 5 to high-risk). EEC12/ stage IA patients had an excellent cause-specific 5-year survival of $98.5 \%$.

Conclusions Pathologists can select cases for p53 testing with high sensitivity and low risk of false negativity. Molecular characterization of endometrial carcinomas has great potential to refine ESGO risk classification for a small subset but offers little value for approximately half of endometrial carcinomas, namely, EEC12/stage IA.

\section{EPV104/\#228 EMERGING IMMUNOTHERAPY PARADIGMS IN ADVANCED ENDOMETRIAL CANCER: THE EFFECT OF ONLINE EDUCATION ON CLINICIAN KNOWLEDGE AND CONFIDENCE}

${ }^{1} \mathrm{G}$ Fisher*, ${ }^{1} \mathrm{~A}$ Furedy, ${ }^{1} \mathrm{~J}$ Vandenbroucque, ${ }^{2} \mathrm{~N}$ Colombo. ${ }^{1}$ Medscape, Medical Education, London, UK; ${ }^{2}$ Instituto Europeo di Oncologia, Department of Gynecology, Milan, Italy

\subsection{6/ijgc-2021-IGCS.174}

Objectives This study determined whether online continuing medical education (CME) could improve the knowledge of oncologists (oncs) and obstetricians/gynaecologists (obs/gyns) regarding the rationale and evidence for immunotherapy paradigms in advanced endometrial cancer.

Methods A 30-minute online video lecture was launched for physicians outside the USA August 2020 with data collected to November 2020. Educational effect assessed with repeatedpairs pre-/post-activity- individual participants serving as own control. 3 multiple-choice, knowledge questions and 1 self-efficacy, 5-point Likert scale confidence question were analyzed. Chi-squared test assessed pre- to post-activity change $(5 \%$ significance level, $\mathrm{P}<.05)$. Magnitude of change in total number of correct responses overall, and for each question, determined with Cramer's V $(<.06=$ Modest, $0.06-0.15=$ Noticeable, $0.16-0.26=$ Considerable, $>.26=$ Extensive $)$.

Results $142 \mathrm{obs} / \mathrm{gyns}$ and 60 oncs completed pre- and postactivity questions. Positive educational effect was observed for obs/gyns (noticeable effect, $\mathrm{V}=.092, \mathrm{P}<.01$; average\% of correct responses increasing from 33 to $42 \%$ ) and oncs (noticeable effect, $\mathrm{V}=.150, \mathrm{P}=.0043$; average $\%$ of correct responses increasing from 47 to $62 \%$ ). Increases in correct responses post-activity seen for questions on response to 2 nd line chemotherapy (\% relative improvement, obs/gyn: 23\%, oncs $22 \%$ ), rationale for immunotherapy (obs/gyns: 24\%, oncs: 72\%), data for the dostarlimab GARNET trial (obs/gyns: 36\%, oncs: 21\%). Confidence in knowledge of the evidence for immunotherapy strategies increased post-activity (total average confidence shift: $27 \% \mathrm{obs} / \mathrm{gyns}$ and $40 \%$ oncs). Overall, $22 \%$ of learners' responses were improved and $39 \%$ of learners' responses were reinforced.

Conclusions This online CME activity resulted in a positive educational impact for both clinical specialties. However, education gaps remained evident post-activity.

\section{EPV105/\#237 COMBINATION TARGETED TREATMENT WITH MEK AND PAN-ERBB INHIBITORS ENHANCES ANTITUMOR ACTIVITY IN ERBB AMPLIFIED EX- VIVO SEROUS ENDOMETRIAL CANCER CELLS}

${ }^{1} \mathrm{~A}$ Larish*, ${ }^{2} \mathrm{~W}$-H Lin, ${ }^{3} \mathrm{~J}$ Smadbeck, ${ }^{3} \mathrm{~S}$ Sotiriou, ${ }^{3} \mathrm{~J}$ Cheville, ${ }^{1} \mathrm{~F}$ Harris, ${ }^{1} \mathrm{R}$ Feathers, ${ }^{1} \mathrm{~J}$ Lynch, ${ }^{4} \mathrm{~A}$ Kumar, ${ }^{5} \mathrm{M}$ Block, ${ }^{1} \mathrm{~L}$ Jones, ${ }^{1} \mathrm{G}$ Karagouga, ${ }^{1} \mathrm{~A}$ Mccune, ${ }^{5} \mathrm{~A}$ Mansfield, ${ }^{3} \mathrm{~S}$ Murphy, ${ }^{4} \mathrm{~A}$ Mariani, ${ }^{3} \mathrm{G}$ Vasmatzis, ${ }^{2} \mathrm{P}$ Anastasiadis, ${ }^{5}$ J Weroha. 'Mayo Clinic, Obstetrics and Gynecology, Rochester, USA; ${ }^{2}$ Mayo Clinic, Department of Laboratory Medicine and Pathology, Jacksonville, USA; ${ }^{3}$ Mayo Clinic, Department of Laboratory Medicine and Pathology, Rochester, USA; ${ }^{4}$ Mayo Clinic, Division of Gynaecologic Surgery, Department of Obstetrics and Gynaecology, Rochester, USA; ${ }^{5}$ Mayo Clinic, Medical Oncology, Rochester, USA

\subsection{6/ijgc-2021-IGCS. 175}

Objectives ERBB pathway alterations present therapeutic targets in high grade endometrial cancer (EC), but efficacy can be limited by persistent co-activation of other ERBB binding partners. The efficacy of dual-inhibition MEK+pan-ERBB or $\mathrm{BET}+$ pan-ERBB in an ERBB2/ERBB3 amplified EC was investigated via $3 \mathrm{D}$ microcancer ex-vivo cell assay.

Methods Tumor was prospectively collected from a patient with stage IIIc1 serous EC. Whole exome, mRNA, and MatePair genomic characterization was performed. Tumor cells were grown in $3 \mathrm{D}$ culture and subjected to titrating drug treatments. Cell viability was determined by the CellTiter-Glo Luminescent Assay. Data transformation and dose-response curves were generated using GraphPad PRISM using the variable slope model. CalcuSyn software with the Chou-Talalay method analyzed drug interactions and synergy. Afatinib, binimetanib, and JQ1 were used to inhibit pan-ERBB, MEK1/2, BET, respectively. For translational relevance, inhibitory effect was defined as percent reduction in ATP from baseline at the
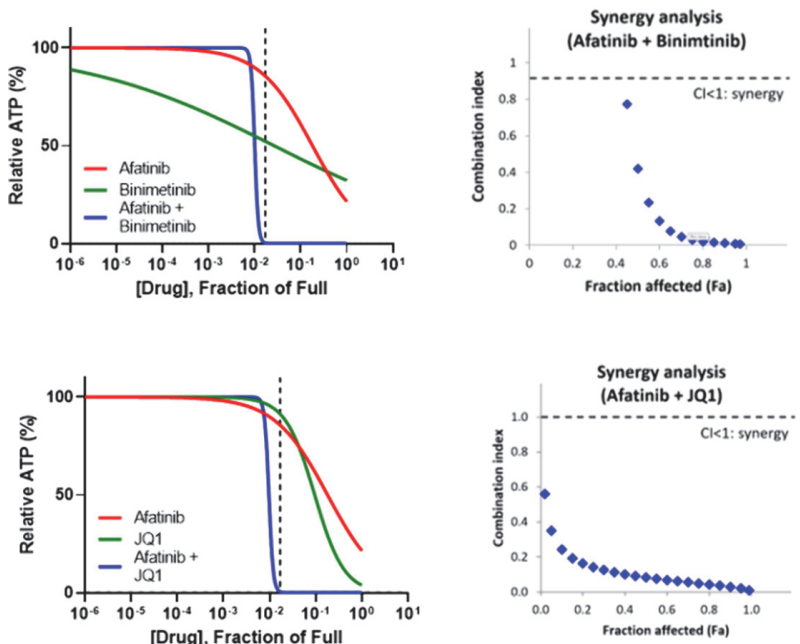

Abstract EPV105/\#237 Figure 1 Microcancer ex vivo exposure to MEK+pan-ERBB inhibitors. Dose response curves of single and combination treatments (left) were 10-fold titrated across 8 log doses for each agent. The highest concentration (i.e. fraction of ful $(\mathrm{FoF})=1$ ) of afatnib, binimetinib and afatinib+JQ1 was $3 \mathrm{uM}, 10 \mathrm{uM}$, and $50 \mathrm{uM}$, respectively. The physiologically achieavable concentration of afatinib is insicated (dotted lines). A comnination index ( $\mathrm{Cl}$, right) was used to assess synergy with afatinib+binimtinib and afatinib+JQ1 as shown by $\mathrm{Fa}-\mathrm{Cl}$ plots. 